\title{
Learning and Cognition in Mathematics
}

\author{
Gaye Williams and Hsin Mei Huang
}

\begin{abstract}
Aims
Learning and cognition is a classical and very vital area in research on mathematics education. Researchers have published many valuable research findings that have contributed to significant development in this area. The continued efforts of researchers now and in the future will, we hope, lead to extensive 'pay-offs'. Different to many other special and related TSGs, such as teaching and learning of algebra, geometry, measurement, statistics, calculus, reasoning, proving and problem solving, to mention a few, TSG22's participants will contribute a more general focus on learning and cognitive activity, and insights into students' characteristics; their strengths and weaknesses in the process of mathematics learning. The TSG focus can include any teaching and learning contexts: from kindergarten to tertiary level, adult education, and teacher professional development. TSG22 discussions should be balanced between theories and their practical applications in mathematics teaching and learning.
\end{abstract}

Organizers Co-chairs: Gaye Williams (Australia), Hsin-Mei Huang (Taiwan), Team Members: Sungsun Park (Korea), Mariana Saiz (Mexico), Jerry Becker (USA); Liaison IPC Member: Shiqi Li (China).

G. Williams $(\bowtie)$

Deakin University, Geelong, VIC, Australia

e-mail: gaye.williams@deakin.edu.au

H.M. Huang

Taipei Municipal University of Education, Taipei, Taiwan

e-mail: hhuang22@gmail.com

(C) The Author(s) 2015

S.J. Cho (ed.), The Proceedings of the 12th International Congress

on Mathematical Education, DOI 10.1007/978-3-319-12688-3_47 


\section{Focus and Themes}

Psychological characteristics of students that influence their inclination to think creatively in mathematics

- Effects of psychological characteristics on students' test performances

- The role of optimism (resilience) in mathematical problem solving Cognitive processing associated with the creative constructing of knowledge

- What aspects of curriculum development/materials contribute to developing learners' mathematical thinking, mathematical inquiry or mathematical creativity?

- What cognitive processes are associated with autonomous student development of new knowledge and what 'teacher moves' can promote such activity?

Mathematical thinking accompanied by affective elements

- In what ways are cognitive, social, and affective elements connected during the development of new knowledge?

- The nature of affective elements that can accompany creative mathematical thinking.

Social interactions associated with creative mathematical thinking

- What aspects of teaching mathematics (teaching behaviors) contribute to developing mathematical thinking, mathematical inquiry or creativity in mathematics?

- What characteristics of classroom interaction or discourse (students-students; teacher-students) facilitate or contribute to knowing mathematics or developing thinking or inquiry abilities?

- What mathematical problems are there that have good use in the classroom by teachers that contribute towards developing cognition in mathematics?

The nature of mathematical understanding

- Children's interpretation of and performance on national and international math tests

- The rationale behind selecting a wrong answer in multiple-choice items in mathematics assessments.

- Contexts for developing mathematical understanding

\section{Number of Submissions and Attendants}

31 papers were reviewed and the following decisions were made: 5 long presentation $(16 \%), 8$ short presentation $(26 \%), 11$ posters $(35 \%)$, Overall acceptance rate was $77 \%$. 
The number of attendants at each session was between 30 and 45 . Each of the four TSG22 sessions attracted a large audience and this added to the stimulating nature of the discussions. With so many thought provoking contributions, and the differences in perspectives communicated, there was insufficient time to pursue all of the interesting questions and issues that arose. The panel's post-ICME communications with participants and others visiting the ICME TSG22 site illustrate ongoing interest and reflections arising from the work of TSG22.

\section{Schedule of TSG22}

\section{Session 1, Tues 10, 10:30-12}

Welcome, Overview

Luis Radford (Invited Plenary), Sensuous Cognition: Mathematical thinking as a Body- and Artifact-based Social Practice (30 min)

Round Table 1: In school and out of school mathematics learning (12 min)

- Paper 1, Michaela Regecova \& Maria Slavickova, How Students' Everyday Experiences Influence Their Mathematical Thinking

- Paper 2, Rankin Graham, Homework: Pre-calculus Algebra Class

- Paper 3, Kadian M. Callahan, Prospective middle School teachers' generalizing actions (reasoning about algebraic and geometric representations)

- Question/Discussion

Poster Session (Parallel to Round Table 1)

Jorge Soto-Andrade \& Pamela Reyes-Santander, Mathematical cognition in young offenders

Shin-Yi Lee (Invited Early Career Researcher), Analysis of "look back" strategies in mathematical problem solving

Hsin-Mei Huang, Children's thinking about measuring areas

Plenary Discussion (4 min)

Session 2: Wednesday, July 11, 10:30-12

Introducing Session

Lianghuo Fan (Invited Plenary), Learning of Algorithms: A Theoretical model with focus on cognitive development (30 min)

Rosa Ma. Garcia \& Mariana Saiz (Electronic), Listening to children explain wrong answers

Terezinha Nunes \& Peter Bryant, Children's' Understanding of Probabilities

Yasufumi Kuroda \& Naoko Okamoto, How can brain activity contribute to understanding of mathematical learning process

Plenasry Discussion (10 min)

Session 3: Friday, July 13, 11-12:30

Introducing Session

Rina Hershkowitz, Tommy Dreyfus, Michal Tabach, Chris Rasmussen, Megan Wawro (Invited Plenary Team) (55 min) 
- Hershkowitz, Dreyfus, \& Tabach, Exponential growth: Constructing knowledge in the classroom

- Chris Rasmussen, Megan Wawro Documenting collective activity in the classroom

- Michal Tabach, Rina Hershkowitz, Chris Rasmussen, \& Tommy Dreyfus, Exponential Growth: Co-ordinating Construction of Knowledge and Documenting Collective Activity in the Classroom

- Question/Discussion

Hong Seek Eng, Lee Ngan Hoe, \& Darren Yeo Jian Sheng, Metacognitive approach: Kick- starting problem solving activity

Gaye Williams (Co-chair) Linking confidence, persistence, and optimistic problem solving activity

Plenary Discussion (13 Mins)

Session 4: Saturday, July 14, 10:30-12

Introducing Session

Alan Schoenfeld (Invited) (30 Mins) Social dynamics for supporting creative mathematical thinking and problem solving

RT2a: Promoting creative thinking: international perspectives (12 min)

- Paper 1, Xianwei Yuan Van Harpen: Creativity and problem posing in US and China

- Paper 2, Yeojoo Jin: Problem solving in Korea

- Paper 3, Cristina Frade, Steve Lerman, Luciano Meira, Peter Winbourne: Working with the ZPD to Identify Learning as Participation in Mathematical Practices

- Question/Discussion

RT2b: Developing understandings of complex mathematical ideas (Parallel to RT 2a) (12 min)

- Paper 1, Revathy Parameswaran: Expert mathematicians approach to understanding definitions

- Paper 2, Megan Wawro: Student reasoning about invertible matrix theorem in linear algebra

- Paper 3, Jun Mun Kyeong Semantic and syntactic reasoning on the learning of algebra

- Question/Discussion

Yuka Koizumi \& Keiko Hino Social interactions of competent teacher: Stimulating creative thinking

Plenary Discussion (11 Mins)

Where to Now? (20 Mins) 


\section{Brief Summary of Outcomes}

The ICME TSG22: Learning and Cognition co-chairs Hsin-Mei Huang and Gaye Williams provide a brief overview of what occurred in preparing for and participating in the ICME-12 TSG22 Learning and Cognition. The TSG22 Panel invited five researchers/research team presentations (Luis Radford; Alan Schoenfeld; The Tommy Dreyfus, Rina Hershkowitz, Michal Tabach, Chris Rasmussen, Megan Wawro Team; The Terezinha Nunes, Peter Bryant Team; and Lianghuo Fan), and two Early Career Researchers (Michal Tabach and Shin-Yi Lee) to highlight cutting edge research in this TSG. The announcement of the 2011 ICMI Awards (Hans Freudenthal Award: Luis Radford; Felix Klein Award: Alan Schoenfeld) contributed further to the interest already shown in this TSG. The quality and number of papers submitted through the reviewing process created dilemmas: how could we enable the sharing of the rich contributions proposed? We decided upon round tables presented simultaneously with many short presentations. Researchers rose to the challenge of showcasing their studies succinctly but with sufficient depth to allow others to follow up on their work. The TSG22 Poster Sessions were well attended and contributed further to TSG22 research.

Some of the connections identified between various presentations are now identified. For example, Radford, and Koizumi and Hino, and Schoenfeld focused differently on 'culture'. Radford on cognition as a 'culturally and historically constituted form of creative responding' with 'sensation considered as a substrate of the mind ...', Koizumi and Hino on the learning culture set up by the teacher to 'stimulate[s] children's creative mathematical thinking', and Schoenfeld on the development of classroom cultures in which 'the students had internalized the relevant mathematical standards' to become 'accountable to the discipline (as opposed to, or in addition to, accountable to the teacher)' and 'able to speak with mathematical authority'. The Hershkowitz Team adapted existing methodological tools to network theories in studying 'the role played by individuals and groups in the class as well as by the class as a whole, in the knowledge constructing process'. Williams examined psychological influences on processes associated with creative construction of new knowledge during problem solving, and Ngan Hoe Lee's Team, and Shin-Yi Lee examined metacognitive processes associated with problem solving. Mathematical understanding and how it develops was explored in probability (by Nunes and Bryant), and in children's developing understandings of area formulae (by Huang). A 'theoretical model for the learning of algorithm with focus on students' cognitive development' was presented by Fan. Soto-Andrade and Reyes-Santander illustrated creative mathematical activity amongst young offenders thus identifying a fruitful area for further research, and Yasufumi Kuroda and Naoko Okamoto's research on brain activity provided a reminder of an expanding area of research in learning and cognition.

The 2014 ZDM Special Edition 'New Perspective on Learning and Cognition in Mathematics Education' (presently under construction) extends many invited presentations and long presentations within TSG22 along four broad themes: 
- Contributions of 'Culture' to Cognition;

- Cognitive, Social, and Psychological Elements of Knowledge Construction;

- Influences of the Mathematics as 'Taught' on Mathematical Thinking and Mathematical Understandings; and

- Focusing Students on Learning Processes Including Problem Solving Processes.

Open Access This chapter is distributed under the terms of the Creative Commons Attribution Noncommercial License, which permits any noncommercial use, distribution, and reproduction in any medium, provided the original author(s) and source are credited. 